\title{
LA TYPOLOGIE DES FOURS CÉRAMIQUES DE DUROSTORUM
}

Crişan Museţeanu

Dan Elefterescu

Adela Bâltâc

L'intérêt à l'égard des ateliers céramiques, et surtout concernant les installations de combustion, les fours autrement dit, a connu dernièrement une intesification significative dans l'Ouest de l'Europe. Une série de travaux parus récemment sur la technique de construction et sur le mode d'emploi élargissent beaucoup notre aire de connaissance en la matière. On est ainsi parvenu, par exemple, jusqu'à pouvoir calculer le volume de pièces traitées dans ces fours et la quantité de bois nécessair à cette fin ${ }^{1}$.

Dans notre zone, et on a en vue ici les provinces du Bas-Danube, les découvertes les plus spectaculaires en ce sens sont faites dans les ateliers céramiques situés autour de la ville de Nicopolis ad Istrum, fouillés par Bogdan Sultov. Malheureusement, la disparition prématurée du regretté archéologue bulgare a enlevé à la recherche archéologique la chance d'une étude plus détaillée sur les fours de Butovo, Pavlikeni et Hotnita, à propos desqueles on ne dispose à présent que de notes assez schématiques ${ }^{2}$. Certes, des découvertes de fours pour des matériaux de construction, de poterie, voire même pouirıdes projectils en argile,ont été, faites en plusieurs points en Dobroudja ${ }^{3}$. En Dacie intra- et extracarpatique, la connaissance des installations à cuire la céramique a progressé considérablement gràce aux découvertes de ces derniers temps. Les fours de cette province sont datés dans une période bien déterminée, à savoir les $\mathrm{II}^{\mathrm{e}}-\mathrm{III}^{\mathrm{e}}$ siécles$^{4}$.

Connue depuis longtemps, la zone des ateliers céramiques de Durostorum comprend, outre les fosses d'extraction de l'argile, un important nombre de fours. Les ateliers produisaient aussi bien des matériaux de construction - qui portent exclusivement des estampilles de la XI legion Claudia que de la poterie d'usage ménager, des lampes et des terres cuites.

Assez lontemps la recherche des ateliers n'a été faite quíncidemment. Des fouilles systématiques ont été entreprises ces derniéres années, 19 fours étant répertoriés. Les recherches seront sans doute poursuivies, mais il est nécessaire et possible, à notre avis, d'essayer dès à présent d'en faire une présentation et d'élaborer une typologie qui soit raccordée à ce que l'on sait en général sur telles installations dans le monde romain.

Du point de vue topographique, les fours se trouvent groupés, à quelques exceptions près (C 19), dans la partie nord-est du site, s'étalant sur environ $400 \mathrm{~m}$ dans la terrasse longeant le

\footnotetext{
1 Vivien Swan, The Pottery KIns of Roman Britain, Londres, 1984; Françoise Le Ny: Les fours de tuiliers gallo-romains, Paris, 1988

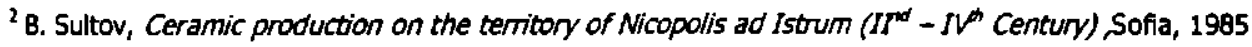

${ }^{3}$ Gh. Ştefan, SCIV, 8, 1957, 1-4, p. 339; A. Radulescu, Monumente romano-bizantine din sectorul de vest al cetăbii Tomis, Constanţa, 1966, p. 16; Idem, Pontica, II, 1969, p. 333; C. Córjan, Pontica, I, 1968, p. 261; A. Panaitescu, Pontica, IX, 1981, p. 304; G. Simion, Peuce, IX, 1984, p. 67; M. Irimia, Pontice, 1, 1986, p. 235; V. Bauman, Peuce, X, 1988, p. 105

'D. Tudor, Oltenia romanz, Bucureşti, 1968, p. 87-88, fig. 18; O. Foca et collabs, Micia, grupul de cuptoare romane de ars ceramica, Deva, 1970; Gh. Popilian, Ceramica romana din Oltenia, Craiova, 1976; Doina Benea, Potaissa, 3, 1982, p. 27; D. Alicu, Acta M.N., XXI, 1984, p. 467; Gh. Bichir, SCTV, 40, 1989, 1, p. 45; I. Stángã, Acta M.N., 34, I, 1997, p. 621

${ }^{5} \mathrm{~N}$. Anghelescu, SCIV, 6, 1955, 1-2, p. 312; C. Museteanu, Dacia, N.S., 29, 1985, 1-2, p. 147
} 
Danube. Certains en ont été détruits par l'écroulement des bords lors de la crue des eaux du fleuve. Leur emplacement fait croire que, depuis les temps anciens jusqu'à nos jours, la configuration du terrain n'a pas trop changé. Nos recherches ont toutefois remarqué dans certaines zones un dépôt assez important de terre, stérile archéologique, de 1,70 m d'épaisseur. Le dépôt date d'après le XI siede, puisquîl couvre un habitat de cette époque-là. Qu'il soit d'origine éolienne on alluvionnaire, ce depôt peut cacher encore d'autres fours. Cette affirmation repose sur la situation présentée par le complexe de fours $C 11, C 16$ et $C 17$. Si le $C 11$ était perceptible dans le bord de l'eau, les $C 16$ et $C$ 17 n'ont fait leur apparition qu'à quelques mètres à l'intérieur, seulement. après une plus ample decapage. Il n'est pas exclu qu'on trouve des situations pareilles dans toute la zone.

Comme déjà dit, le nombre de fours était sans doute plus grand; notre discussion ne porte pourtant que sur les 19 complexes ayant bénéficié d'une minime recherche, même si certains sont dans un état de dégradation avancée et leurs éléments de construction ne sont pas tous saisissables. Pour leur analogies morphologique nons avons adopté des systémes typologiques employés ordinairement dans les eutres provinces de l'Empire. En essence, on prend pour point de départ la forme du foyer, circulaire ou rectangulaire ${ }^{6}$. Quand aux termes employés pour désigner chaque élément du four, nous avons adopté la terminologie de Françoise Le Ny et Vivien Swan', qui est en ses grandes lignes, très proche de celle employée dans les provinces du Bas-Danube, respectivement la Dacie et la Mésie Infèrieure ${ }^{8}$ (fig.1)

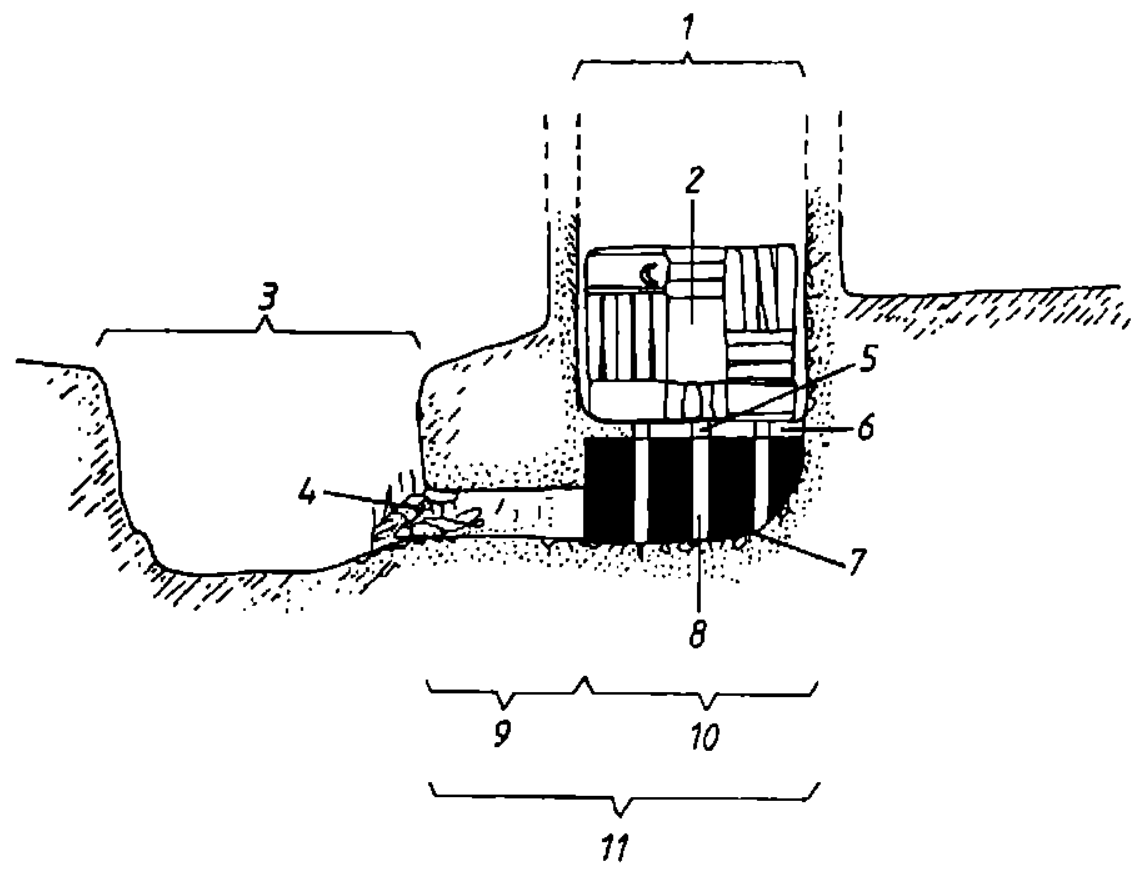

Fig. 1. 1. Laboratoire, 2. Charge a cuire, 3. Aire de chauffe, 4. Gueule, 5. Comeaux, 6. Sole, 7. Murets de soutement, 8. Conduits de chateur, 9. Alandier, 10. Chombre de chauffe, 11. Foyer

\footnotetext{
${ }^{6}$ P. Duhamel, Acta Praestorica et Archeologica, Berlin, 9/10, 1979, p. 63, fig.34

${ }^{7}$ Ibidem, 0.54, fig.5; Françoise Le Ny, op.cit., p. 20, fig. 5

${ }^{\circ}$ Gh. Popilian, op.ct, p. 140; B. Sultov, op.cit., p.39
} 
Les fours de Durostorum sont:

- à foyer circulaire

- à foyer rectangulaire

I La première catégorie, plus nombreuse, compte 14 complexes: C 1-6, C 8?, C 11-17.

L'analyse morphologique s'occupe ensuite des types de support de la sole. Pour l'instant, à Durostorum il n'y a attestée que celle à pilier central. En principe, fait exception le C 8, mais son mauvais état de conservation et sa recherche périégétique rendent la determination difficile?. Comme il s'agit d'un four pour la cuisson de vases, nous pensons à le placer dans ce groupe.

Les complexes des groupe I de Durostorum se présentent ainsi:

Le foyer est creusé dans la terre vierge, les parois verticales portent un enduit qui peut être à plusieurs couches. Les parois sont fort brûlées, parfois jusqı' à la vitrification. L'épaisseur de la brûlure varie de 0,04 à $0,08 \mathrm{~m}$. Les foyers sont de dimensions sensiblement différentes, leur diamètre étant de 0,30-0,45 m. La gueule large d'environ $0,25-0,30 \mathrm{~m}$ est de règle orientée vers le nord, c'està-dire vers le Danube, à deux exception connues jusqu'ici: $C 11$, orientée vers le sud et $C 16$, vers le nord-est. Dans certains cas, en tant que senil de la gueule on trouve un fragment de brique $(0,26 x$ $0,22 \mathrm{~m}$ pour le $\mathrm{C}$ 13). Le pilier au centre du foyer est construit de deux façons: a) de la terre ménagée au moment de l'aménagement ( $C 4-5, C 16)$; b) en briques $(0,30 \times 0,15 \mathrm{~m}$. - C1) à liant d'argile. Le pilier ainsi construit est enduit tout autour $(\mathrm{C} 1, \mathrm{C12}-13)$. Son diamètre mesure entre $0,30 \mathrm{~m}$ à $0,44 \mathrm{~m}$. La gueule était dans certains cas renforcée (C11) avec deux tubes de canalisation placés d'une part et de l'autre; une tuile fermes cette bouche.

Un canal vertical partait parfois de la gueule et était plaqué de tuiles disposées verticalement $(0,30 \times 0,15 m-C 1)$.

Devant le canal it y avaient un aire de chaufage sous la forme une fosse (C17) aux dimensions de $1,57 \times 1,40 \mathrm{~m}$ et profond de $0,38 \mathrm{~m}$.

La sole qui supporte le laboratoire de forme circulaire et aux dimensions du foyer est construite en briques $(0,30 \times$ $0,15 \mathrm{~m}$ ) à liant d'argile. L'épaisseur de la sole varie de 0,10 à $0,14 \mathrm{~m}$. Des carneaux, disposés en cercles concentriques, (au diamétre de 0,05-0,07 m) perforaient la sole en divers endroits. Dans la plupart des cas le laboratoire manque, ayant été détruit dès les temps anciens. Seuls quelques fours C4 (fig. 2), C5, C11 et C17 conservent des restes de parois du laboratoire. L'étude de ceux-ci permet certaines considérations sur la façon dont était construit cet espace. Il y en avait deux modalités:

1. Les parois du laboratoire étaient en torchis à enduit d'argile. A l'extérieur le même enduit (C4, C12 et C17).

2. Les parois du laboratoire étaient bâties en briques à liant d'argile, également enduites d'argile à l'intérieur $(\mathrm{C} 5, \mathrm{C} 11)$.

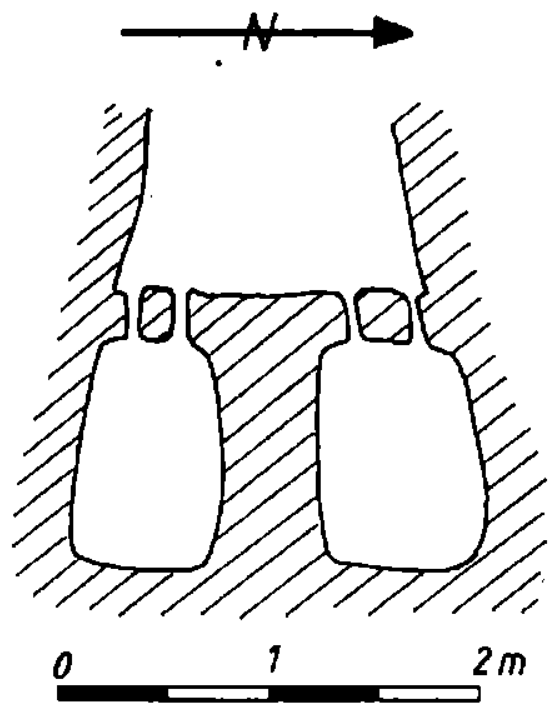

Fig. 2. Four 4

Le niveau auquel ont été trouvées les parois ne saurait nous offrir des informations sur le mode de fermeture de cette chambre. L'inclination des parois

${ }^{9}$ D. Elefterescu, Culturà și civilizatje la Dunårea de Jos, I, 1985, Călărasi, p. 83 
indique le fait que la partie supérieure du laboratoire étaint voûtée. Compte tenu des analogies, il est à supposer qu'une ouverture existait dans la partie supérieure de la voûte, par laquelle étaient introduites les vases et par où on réglait le tirage.

Typologiquement parlant les fours sont bien connus en dépit de toutes les différences de détail que présente leur construction. C'est que leur origine et leur durée dépassent le monde romain $^{10}$. Rappelons le fait que ont été déconverts des fours de ce type à l'époque impériale pratiquement dans toutes les provinces, à commencer par Britannia, ensuite Hispania, Gallia, Germania et Pannonia ${ }^{11}$ et ainsi de suite.

La forme est présente tant au nord du Danube, en Dacia ${ }^{12}$, qu'au sud du fleuve, dans le périmètre de la Mésie Inférieure ${ }^{13}$.

Du point de vue de la chronologie, on doit reconnaître qu'au niveau actuel de nos connaissences on ne saurait parler d'une datation exacte, ce type de four ayant une large utilisation qui va au-delà des limites temporelles du monde romain. On peut toutefois en appeler à la chronologie générale des ateliers céramiques de Durostorum et, là où la chance nous a aidés, à la datation du complexe à travers les matériaux archéologiques, découverts dedans ou aux alentours. D'après les matériaux découverts dans le périmètre des ateliers, le moment où ce four a fait son apparition se situe au début du $\mathrm{II}^{\mathrm{e}}$ sièie et son utilisation prend fin dans la première ou la seconde partie du IV siècle $^{14}$. Sans doute, certains complexes dont on discute peuvent être datés avec plus de précision. Ainsi, par example, $\mathrm{C} 1$ (éventuellement $\mathrm{C} 3$ aussi, selon les affirmations de son découvreur), peut être daté, en base de la céramique existente, au II $^{\mathrm{e}}$ siècle ${ }^{15}$. De même, C11 où l'on a trouvé des produits de rebut, les vases peuvent être datées du $\mathrm{II}^{\mathrm{e}}$ siècle, sa seconde moitié probablement ${ }^{16}$. La lampe cordiforme de $\mathrm{C} 16$ indique une datation similaire. D'ailleurs, entre ces deux derniers complexes qui emploient le même canal d'alimentation il y a en probablement succession. C'est le C16 qui a fonctionné d’abord et après sa destruction et son nivellement, a été construit le C11. Près du C12 on a également découvert des pièces céramiques. Il s'agit, entre autres, d'un moule de terrecuite datable aux II $^{e}-$ III $^{e}$ siècles. La charge de vases de C8 date également du II $^{e}$ siècle. On peut dire que la plupart des fours de ce type découverts jusque là, et qui peuvent être datés, sont du II $I^{\mathrm{e}}$ siècle.

La seconde catégorie réunit des four de forme rectangulaire. À Durostorum n'ont été fouillés jusqu'à présent que quatre fours de ce type, lesquels relèvent de trois sous-groupes différents; en raison des différences de construction du foyer.

II a. Four rectangulaire à un seul canal médian. En font partie les $\mathrm{C} 7$ et $\mathrm{C} 10$, qui sont des fours de grandes dimensions.

\footnotetext{
${ }^{10}$ Ninetta Cuomo di Caprio, Sibrium, 1972, p. 410, II, fig.II; R.Hampe, A.Winter, Bej Topferinen Zieglen in Süditelien, Sizilien und Griechenland, Mainz, p. 30-31, fig. 21, 1965; P. Duhamel, op. cit, p. 58, fig. 5 et fig. 31

11 M.R. Hull, The Roman Potters Kins of Colchester, Oxford, 1963, fig. 4; M.I. Jiménéz Cisneros, Germania, 36, 1958, p. 474, pl. 67, nos. 1-3; P.La Baume, Germania, 37, 1959, p. 293-296

12 O. Foca et collabs, op. cit., p. 38, fig. 16-17; D. Tudor, op. cit., p. 87-88, fig. 18; Gh. Popilian, op.cit.,p. 140; H. Daicoviciu et collabs, Materiale sji cercetdri ameologice, Tulcea, 1980, p. 282; Doina Benea, op. cit., p. 27; I. Stanga, op. cit.,p. 621

${ }^{13}$ B. Sultov, op. cit., p. 40

${ }^{14} \mathrm{C}$. Muşeţeanu, op. cit., p. 150

$15 \mathrm{~N}$. Anghelescu, Pontica, 4, 1971, p. 289

${ }^{16}$ C. Muşeţeanu et collabs, Cronica cercetarilor ameologice campania 1996, Bucureşti, 1997, p. 43
} 
En général, les fours sont conservés jusqu'à la hauteur de sole; la gueule et le foyer sont construits de la terre vierge. Suite à la cuisson, une couche de terre de couleur rougeâtre, fortement brûlée, mesure environ 0,25 m. Les parois intérieures portent un enduit d'environ 0,02 m.

Le foyer est construit en voûte, avec des tuiles à liant d'argile. Le canal médian, en voûte lui aussi à l'aide de tuiles, arrive jusqu'au fond du four; à partir de ce canal, cinq parois latérales, en terre ménagée, forment des canaux dérivés.

La sole (conservée partiellement) est formée de couches successives d'enduit d'argile, de torchis et de fragments de briques (fig. 3). Le laboratoire, absent, pouvait être construit de piles de briques ou de tuiles dressées directement sur la sole, les parois extérieures étant faites d'un encollage d'argile. L'absence de toute trace de parois, que ce soit en argile ou de briques, nous fait pencher vers cette hypothèse qui trouve des analogies ailleurs dans l'Empire.

La description de l'installation a été faite d'après le C10 (fig. 4), le mieux gardé de dégradation, mais ses composants conservés semblent indiquer une typologie identique ou semblable à celle du $C 7$. Considéré le plus souvent comme spécifique pour la cuisson des briques et des tuiles, ce type de four se retrouve dans nombre de centres importants du monde romain ${ }^{17}$. À titre d'exemple, nous avons choisi quelques fours de ce type d'une très large bibliographie. Ils sont signalés dans les provinces: Gallia, Germania, Britannia, Pannonia et Dacia ${ }^{18}$.

Par sa forme, le C7 se rapproche - à part certaines différences de détail - d'un four découvert à Dinogetia ${ }^{19}$, qui est daté de la fin du III ${ }^{\mathrm{e}}$ siècle et le début du siècle suivant. Pour l'instant on manque de tout élément de datation pour ce type de four à Durostorum. De toute

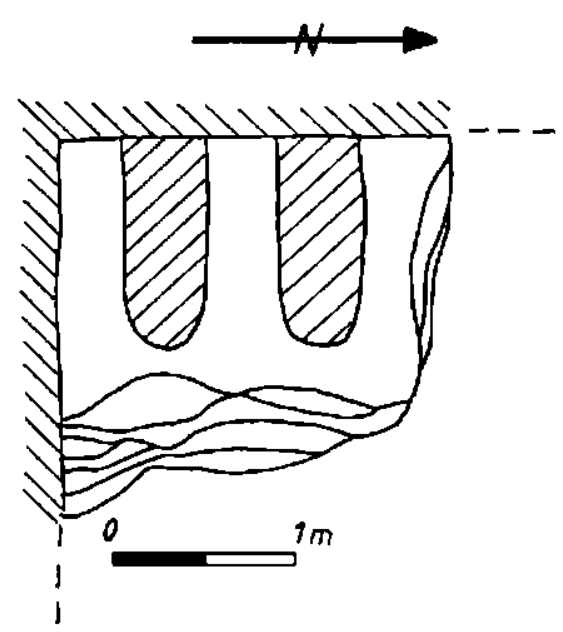

Fig. 3. Four 7 façon, en nous rapportant à la durée d'existence des objets que nous supposons avoir été cuits lá, il peut être situé dans les II ${ }^{\mathbf{e}}$ - IV siècles.

II b. Four de forme rectangulaire à paroi médiane. C9 est la seule installation à faire partie de ce groupe. Le foyer creusé dans la terre vierge est partagé en deux couloirs par une paroi médiane construite de briques $(0,36 \times 0,40 \mathrm{~m}$.) à liant d'argile; la paroi du fond du foyer n'est pas plaquée de briques. La sole est en briques à liant d'argile sur lesquelles on a appliqué une couche d'enduit; l'epaisseur en est de 0,14 m. Les carneaux ( $\emptyset=0,08-0,10 \mathrm{~m}$.), sont disposés à $0,35-0,40 \mathrm{~m}$. d'intervalle l'un de l'autre, sur trois rangées. La voûte du laboratoire est écroulée sur la sole depuis le niveau de 0,55 m. Le laboratoire était construit de briques liées par une couche épaisse d’argile (fig.5).

\footnotetext{
${ }^{17}$ Ninetta Cumo di Caprio, op. cit., p.429, type II b; F.Le Ny, op. cit, p. 15, fig. 3 b, II c

${ }^{18}$ F.Le Ny, op. cit, p. 33; F. Reutt, Germania, 61, 1983, 1, p. 53; M. R. Hull, op. cit., p. 3, four 7, fig. 4; B. Kuzinski, Budap. Règ. XI, 1932, p. 27, fig. 4; I. Paulovies, ArchErt, 47, 1934, fig. 100-101; O. Foca, op.cit., p. 82; Gh. Popilian, Dacia, N.S.,20, 1976, p. 228, fig.5/2; Gh. Bichir, op.cit.,p.53, fig. 4; D. Alicu, op.cit.,p. 467

${ }^{15} \mathrm{Gh}$. Stefan, op. cit, p. 339.
} 
Cette forme est elle aussi bien connue dans les ateliers romaines, étant signallée dans l'Ouest de I'Europe, en Italie et dans les proviaces, Gallia, Hispania, Britannia et Germania?. qui est des provinces de notre proximité, des fours de ce genre apparaissent à Aquincum en Pannonie, à Micia et à Romula en Dacie ${ }^{21}$. Dans la Mésie Inférieure on la retrouvé à Novae, mais aussi à Tomis et à Altinum ${ }^{22}$ avec, certes - une série d'éléments de détails différents. Les indices pour dater ce type de four font défaut. Aussi une attribution large aux II $^{\mathbf{e}}-\mathrm{IV}^{\mathbf{e}}$ siécle est - elle la seule possible pour l'instant.

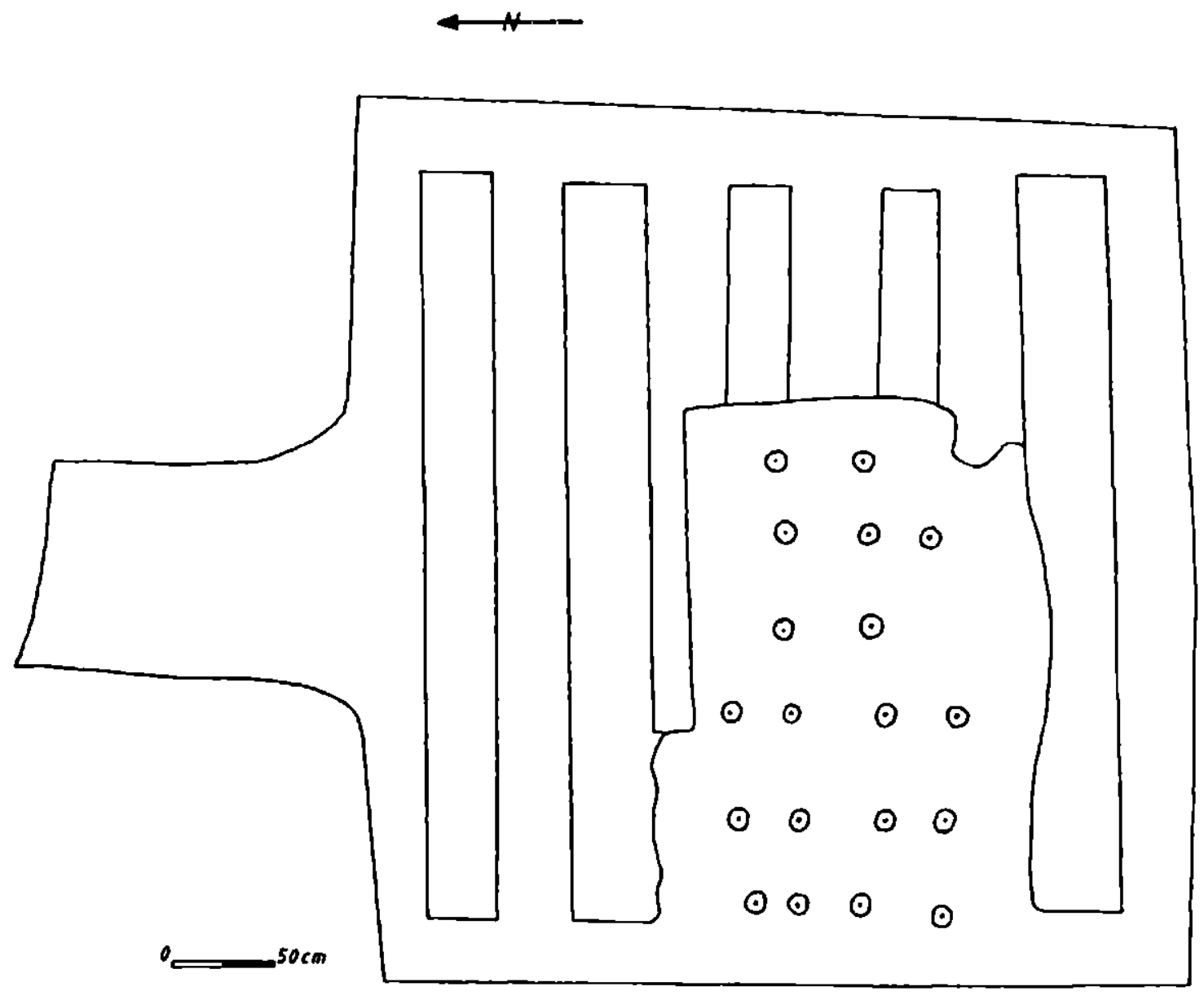

Fig. 4. Four 10

\footnotetext{
${ }^{20}$ Fr. Le Ny, op. clt., p. 15, fig. 3b, Ila; N. Cuomi di Caprio, op. cit., p.435, tip Ilc; M. Sotomayor, Noticiario Arch. Hispanico, B 9, 1946 - 1965, p. 193 - 199, fig. 3 - 4; K. Stade, ORL, Bd. III, Lf g. XLIX, 1933, p. 32 - 33, pl. I, m 1,5; M. R. Hull, op. cit, p. $147-148$, fig. 183 , cuptorul 23 .
}

${ }^{21}$ B. Kurvinsto, op. cit, p. 43, fig.24, p. 44, fig. 25; O. Hoca, op. ct, p.23; Gh. Popilian, Ceramica romana din Ohenia, p. 143, tip 4.

${ }^{2}$ v. Velkov, Archeologija, Sofia, 7, 1966, 1, p. 46; A. Rådulescu, op. cit., p. 342, tip IV; M. Irimia, op. cit, p. 42. 


\section{II c.Four rectangulaire à pilier central pour soutenir la sole (fig. 10).}

Découvert depuis peu, C 19 est la seule installation qui peut être encadrée dans ce soustype. Il présente une nouveauté quant à la modalité de fixer la sole à l'aide de poutres d'argile cuite disposées radialement autours du pllier central. L'élément de résistance du pilier est un tube d'aqueduc de grandes dimensions $(0,80 \times 0,24 \mathrm{~m})$ enterré dans le plancher du foyer jusqu'à $0,20 \mathrm{~m}$. Autour du tube a été dressée une boitte rectangulaire de grands fragments de tuiles, remplie de terre. À la partie supérieure, le tube était couvert par un fragment de tuile. Le pilier entier était couvert par une couche d'argile, sont de profil demi-circulaire. Montées sur le chant, leurs bouts sont appuyés sur les parois. Entre les poutres ainsi montée, des ponts de liaison ont été créés du même matériel. Les parois du laboratoire subsistent jusqu'à la hauteur de $0,20-0,28 \mathrm{~m}$, elles étant comriue le foyer, réalisées en terre ménagée. Ce systéme de poutres est assez bien connu en Occident (Britannia et Germania), mais aussi dans la Méditerranée de l'est, en Créte ${ }^{23}$. Dans la zone des provinces danubiennes, bien que rarement employé, il est présent, selon nos informations, au nord de la Dobroudja, à Teliţaa ${ }^{24}$, daté à la fin du III ${ }^{e}$ au début du IV siècle.

Ce type de four trouve moins d'analogies en Occident. En revanche, au Bas-Danube il est présent à Pavlikenie, où il est employé à la cuisson de la poterie, tout connue chez nous, ainsi qu'à Tomis, où il s'agit du traitement des matériaux de construction ${ }^{25}$. En raison du matériel trouvé sur la sole, l'installation de Durostorum peut être datée du II siècle.

La question qui préoccupe les spécialistes est de savoir quel est le raport entre la forme et la destination des fours. Il est fort possible, sans qu'il y ait règle, que les instalations circulaires, qui sont de tout façon de moindres dimensions, aient été employée à la cuisson de la poterie, des terre-cuites et des lampes, les fours rectangulaires, beaucoup plus grands, étant destinés aux matériaux de construction et aux vases de grandes taille ${ }^{26}$. Les résultats de Durostorum paraissent confirmer, en général, cette hypothèse.

Les fours circulaires découvertes à Durostorum sont, d'après la circonférence $\left(0,44-3,80 \mathrm{~m}^{2}\right)$, de dimensions petites et moyennes. Là où il $\mathrm{y}$ a eu des matériaux, à líntérieur ou dans la proximité $(\mathrm{C} 1, \mathrm{C} 8, \mathrm{C} 13, \mathrm{C} 16, \mathrm{C} 17)$, il s'agissait de vases, de lampes
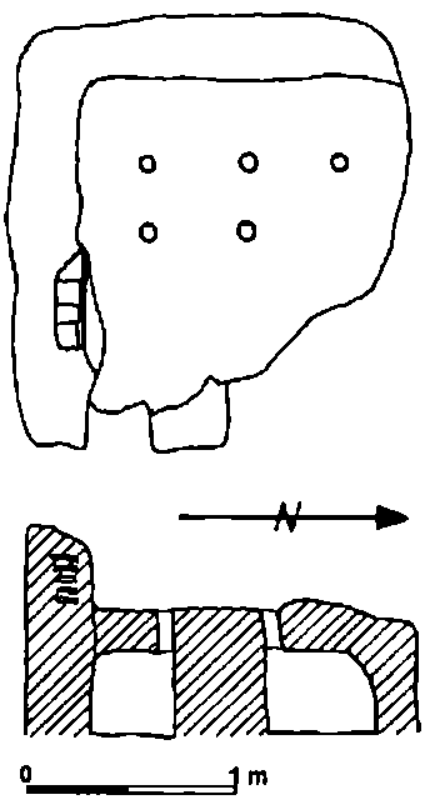

Fig. 5. Four 9 ou de terres-cuites. Le $C 9$, de forme rectangulaire, se rapproche, comme superficie $\left(1,95 \mathrm{~m}^{2}\right)$ des fours circulaires. Il n'a livré malheureusement aucun indice quant aux matériaux qui lui étaient destinés. Bien que de forme rectangulaire, le C19 conservait sur la sole les restes d'une charge de vases. Par contre, le $\mathrm{C} 7$ et surtout le $\mathrm{C} 10$, de grandes dimensions (près de ce dernier il y avait aussi des tuiles), semblent indiquer le fait qu'on les avaient utilisés à la cuisson des matériaux de construction. Les recherches futures apporteront sans doute de nouvelles informations

\footnotetext{
${ }^{23}$ Vivien Swan, op. cit., p. 62-63; BJ CVII, 1901, p. 221-222; R. Hampe, A. Winter, op. cit, p.24, pi. 21

${ }^{24}$ G. Simion, Peuce, IX, 1984, p. 68-69, pl. II, fig. 1,2; pl. IV, fig. 1; V. H. Bauman, Asezari rurale antice in zona Gurilor Dunanii, Contribubi ameologice la cunoasterea habitatului rural (sec I-JV p. Chr.), Tulcea, 1995, p. 296

${ }^{25}$ B. Sultov, op. cit., p. 40, pl. XII,5; A. Rădulescu, OP. cit., p. 245, pl. III

${ }^{25}$ A. Radulescu, op. cit., p. 248; B. Sultov, op. cit., p. 235
} 
là-dessus. On remarque toutefois que le nombre des fours de forme circulaire pour la cuisson des vasses est bien supérieur à celui des fours rectangulaires, employés en général au traitement des briques et des tuiles.

Les moments d'essor constructif impliquent la demande de matériaux en grandes quantités. En analysant, ailleuts, la chronologie des estampilles de la XI legion Claudia découvertes dans notre zone et donc produites dans les ateliers de l'endroit ${ }^{27}$, nous avons identifié plusieurs périodes d'activité soutenue en ce sens. Une première période se situe au début du $\mathrm{II}^{\mathrm{e}}$ siècle, en même temps que l'édification du camps légionnaire; l'étape suivante est de la seconde moitié du siècle, probablement àprès les attaques des Costoboces de 170. Au temps de Caracalla on enregistre un nouveau record de la production de briques et, enrîn, à la fin du III ${ }^{e}$ siècle et au dèbut du siècle suivant, à l'époque donc de Diocletian-Constantin, on y connu là une dernière grande étape constructive. On pourait croire, sans trop risquer de se tromper que le nombre des fours destinés à la cuisson des matériaux de construction était plus important dans ce temps-là. Les èlèments de datation pour le type II a et b de fours appaîtront probablement au cours des futures recherches.

Les données detenues jusqu'ici sur les fours de Durostorum montrent que du point de vue typologique ceux-ci font partie de la série large des installations pour la cuisson de la céramique connues dans le monde romain. Il existe, certes, des détailles différents d'un four à l'autre qui parlent, supposons nous, de l'habilité des potiers plutôt que de principes constructifs divers. On remarque d'ailleurs facilement que les artisans de Durostorum fabriquaient des produits spécifiques dans tout le monde romain, suivant la tendance - déjà connue- à la standardisation.

\section{CATALOGUE}

Abréviations: $L=$ largeur, $H=$ hauteur, $E=$ épaisseur, $\emptyset=$ diamètre, $S=$ surface du four.

Four no. 1: circulaire à pilier central, à l'état fragmentaire. Le foyer (fragmentaire) fait de plusieurs couches d'argile fortement brûlées. La gueule orientée vers le $\mathrm{N}$. Le pilier (fragmentaire, $\mathrm{H}=$ $0,45 \mathrm{~m}$ ) en briques $(0,30 \times 0,15 \mathrm{~m}$ ) à liant d'argile. La sole (fragmentaire) en briques à liant d'argile $(g=0,15 \mathrm{~m})$. Le laboratoire détruit. Sur la sole on a trouvé des vases qui peuvent être reconstitués, quelques-uns à figures, trois moules de lampe (N. Anghelescu, SCIV, 6, 1955, 1-2, P. 312) $1955,1-2$, P. 312)

Four no. 2: circulaire à pilier central(?). Conservé partiellement. (N. Anghelescu, SCIV, 6,

Four no. 3: circulaire à pilier central (?) détruit. (N. Anghelescu, SCIV, 6, 1955, 1-2, P. 312)

Four no. 4: circulaire à pilier central. Conservé partiellement. $S=3,140 \mathrm{~m}^{2}$. Le foyer (fragmentaire, $\emptyset=2,00 \mathrm{~m}$ ) creusé dans la terre ménagée. La gueule orientée vers le $\mathrm{N}$. Le pilier (fragmentaire, $\emptyset=0,50 \mathrm{~m} ; \mathrm{H}=1,00 \mathrm{~m}$ ) construit en terre ménagée. La sole (fragmentaire, $\emptyset=1,50$ m) en torchis. Le laboratoire (fragmentaire) $\emptyset=0,88 \mathrm{~m}$. (C. Muşeţeanu, Dacia, N.S., XXIX, 1-2, 1985, p. 148 , fig. 2).

Four no. 5: circulaire à pilier central. Conservé partiellement. $S=3,801 \mathrm{~m}^{2}$. Le foyer fragmentaire. La gueule orientée probablement au N. Le pilier façonné en terre ménagée. La sole

${ }^{27}$ C. Muşeţeanu, The Roman limes at the Lower Danube $\left(4^{\phi}-\sigma^{\text {th }}\right.$ Century A.D.), Sympozion Murighiol/Halmyris, 1998, p. 169 
(fragmentaire, $\emptyset=2,20 \mathrm{~m}$ ) construite de briques à liant d'argile. Le laboratoire (fragmentaire $\emptyset=2,20$ $\mathrm{m}$ ) en briques (trouvées écroulées à l'intérieur du four). (C. Muşeţeanu, Dacia, N.S., XXIX, 1-2, 1985, p. 148).

Four no. 6: circulaire à pilier central. Conservé partiellement. $S=2,835 \mathrm{~m}^{2}$. Le foyer (fragmentaire) modelé dans la terre ménagée. La gueule orientée vers le $N$ (?). Le pilier (fragmentaire, $\mathrm{H}=1,40 \mathrm{~m}$ ) de terre ménagée. La sole (fragmentaire, $\emptyset=1,90 \mathrm{~m}$ ) construite de briques à liant d'argile. Le laboratoire fragmentaire. (C. Museteanu, Dacia, N.S., XXIX, 1-2, 1985, p. 148 - 149).

Four no. 7: rectangulaire à parois médianes, conservé partiellement. $S=4,64 \mathrm{~m}^{2}$.Le foyer (fragmentaire, $\emptyset=2,00 \mathrm{~m}$ ) creusé dans la terre ménagée. La gueule orientée vers le $\mathrm{N}$. Les deux parois latérales (fragmentaires) élevées de terre ménagée, sont renforcées par cuisson. La sole et le laboratoire sont détruites. (C. Muşeţeanu, Dacia, N.S., XXIX, 1-2, 1985, p. 149, fig. 3).

Four no. 8: circulaire (?).La gueule orientée vers le $N$ (?). La sole détruite. Le laboratoire écroulée. Dans le four ont été trouvés des couvercles et de petits tessons céramiques datés de la seconde moitié du II siècle. (D. Elefterescu, Cultura si civilizatie la Dunarea de Jos, 1, 1985, p. 81-82).

Four no. 9: rectangulaire, à paroi médiane. $S=1,95 \mathrm{~m}^{2}$. Le foyer aux parois latérales en brique $(I=1,20 \mathrm{~m} ; E=0,30 \mathrm{~m})$ à liant d'argile; la paroi au fond du four n'est pas plaquée de briques. La gueule orientée vers le $N$. Le parois $(g=0,04 \mathrm{~m} ; \mathrm{H}=0,50 \mathrm{~m} ; \mathrm{l}=0,40 \mathrm{~m})$ de briques à liant d'argile. La sole (fragmentaire, $E=0,14 \mathrm{~m}$ ) construite de briques à liant d'argile, sur lesquelles on a appliqué un enduit; les carneaux $(\emptyset=0,08-0,10 \mathrm{~m})$ sont disposés par trois rangées et distants de 0,35-0,40 m. Le laboratoire (écroulé) était en torchis ( $\mathrm{I}=1,50 \mathrm{~m}, \mathrm{~L}=1,30 \mathrm{~m} \mathrm{H}$ conservée $=0,55 \mathrm{~m}$ ).

Four no. 10: rectangulaire, à paroi médianes $(L=5,50 \mathrm{~m}, 1=4,14 \mathrm{~m}) . \mathrm{S}=22,72 \mathrm{~m}^{2}$. Le foûyer (en bon état) construit de terre ménagée. Le canal médian $(3,50 \times 3,30 \mathrm{~m})$ voûté avec tuiles. $\mathrm{L}$ a gueule orientée vers le $\mathrm{N}(\mathrm{L}=1,40 \mathrm{~m}, \mathrm{I}=1,34 \mathrm{~m}, \mathrm{H}=0,80 \mathrm{~m})$. Les cinque parois latérales(en bon état, $L=3,50 \mathrm{~m}, \mathrm{l}=0,40 \mathrm{~m}, \mathrm{H}=1,20 \mathrm{~m}$ ) construites de terre ménagée. La sole (fragmentaire) réalisée de couches successives d'enduit d'argile, de torchis et de briques. Le laboratoire détruite(Cronica sãpãturilor arheologice din campania 1993, Satu-Mare, 1994, p. 46).

Four no. 11: circulaire à pilier central, à deux phases de fonctionnement. $\mathrm{S}=32,97 \mathrm{~m}^{2}$ (phase a), $\mathrm{S}=$ $25,12 \mathrm{~m}^{2}$ (phase b). Le foyer (en bon état) construit de terre ménagée. Dimensions: phase $a=1,50 \times 2,00 \mathrm{~m}$, phase $\mathbf{b}=1,20 \times 2,00 \mathrm{~m}$. La gueule orientée vers le S-E (le même canal que $\mathrm{C} 16)$. La gueule du four fermée par une brique $(\mathrm{L}=0,25 \mathrm{~m}, \mathrm{I}=0,38 \mathrm{~m}, \mathrm{H}=0,22 \mathrm{~m})$. Le pilier (entier, $\mathrm{H}=0,60 \mathrm{~m}$ ) est en terre ménagée. La sole (fragmentaire, $L=1,00 \mathrm{~m}$ ) en briques à liant d'argile. Le laboratoire (fragmentaire, $L=2,10 \mathrm{~m}$ - phase $\mathbf{a}, L=1,66$ $m, E=0,10-0,40 m-$ phase $b$ ) construit de torchis (phase a), de briques $(0,20 \times 0,18 \mathrm{~m}$ ) à liant d'argile (phase b). Sur la sole on a trouvé une charge. (Cronica sãpãturilor arheologice din campania 1993, Satu-Mare, 1994, p. 46).

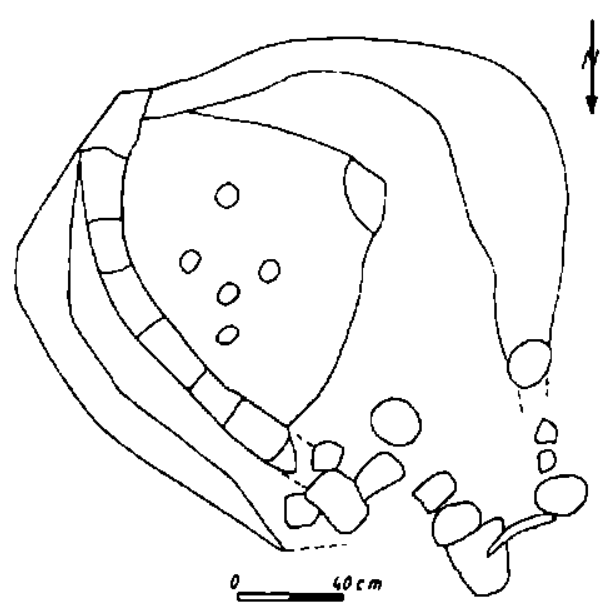

Fig. 6. Four 11 Fig. 6 


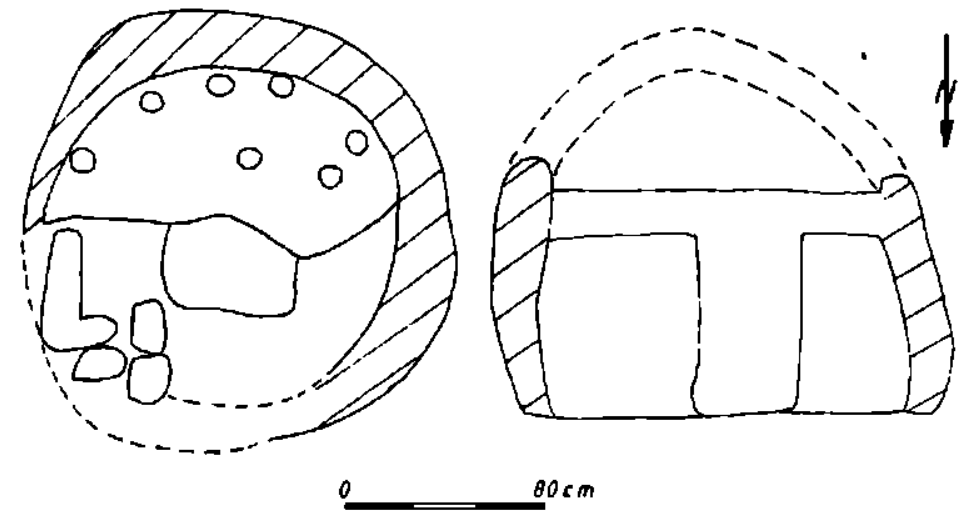

Fig. 7. Four 12

Four no. 12: circulaire à pilier central conservé partiellement.. $\mathrm{S}=0,95 \mathrm{~m}^{2}$. Le foyer (fragmentaire, $\varnothing=1,10 \mathrm{~m}$ ) construit de torchis, fortement brûlé, les parois $E=0,10 \mathrm{~m}$. La gueule orientée vers le $\mathrm{N}$. Le pilier (en bon état, $H=0,60 \mathrm{~m}, E=0,30 \mathrm{~m}$ ) construit de briques et d'argile. La sole (fragmentaire, $\emptyset=1,10 \mathrm{~m}, \mathrm{E}=$ $0,12 \mathrm{~m}$ ) formée de couches, de briques étroites et d'argile, les carneaux $\emptyset=0,70 \mathrm{~m}$. Le laboratoire (fragmentaire, $\emptyset=1,10 \mathrm{~m}$ ) en torchis, les parois $\mathrm{E}=0,16-0,20 \mathrm{~m}, \mathrm{H}=0,38 \mathrm{~m}$. (Cronica sãpãturilor arheologice din campania 1994, Cluj-Napoca, 1995, p. 66). Fig.7, 9 (reconstitution)

Four no. 13: circulaire à pilier central.. $S=0,44 \mathrm{~m}^{2}$. Le foyer (fragmentaire, $\emptyset=0,70 \mathrm{~m}$ ) est de torchis, l'intérieur enduit d'argile, les parois $\mathrm{E}=0,14 \mathrm{~m}$. La gueule orientée vers le $\mathrm{N}$, marquée

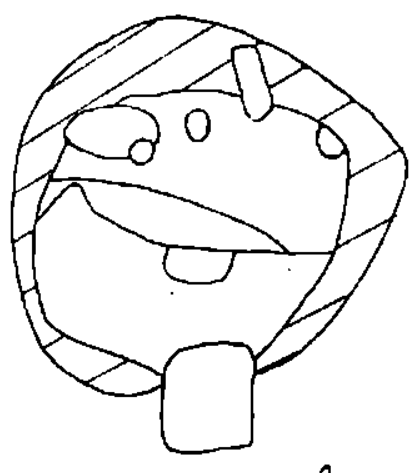

0

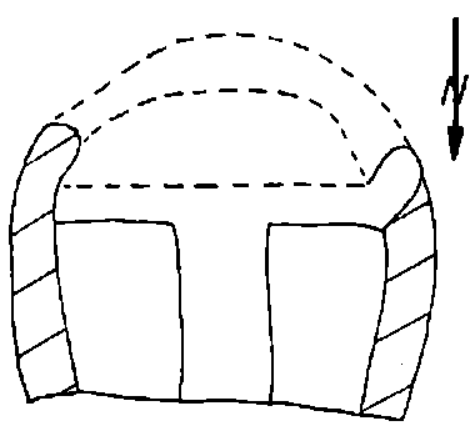

$80 \mathrm{~cm}$

Fig. 8. Four13

à la partie inférieure par une brique $(0,26 \times 0,22 \mathrm{~m})$ formant un seuil. Le pilier (en bon état) fait de briques et de tuiles à liant d'argile $(\mathrm{H}=0,44 \mathrm{~m}$, $E=0,20 \mathrm{~m}$ ). La sole (fragmentaire) formée de couches successives de briques et de tuiles à liant d'argile ( $\varnothing$ $=1,10 \mathrm{~m}$ ), les carneaux ont $\emptyset=0,60$ m. Le laboratoire (fragmentaire, $\emptyset=$ $0,75 \mathrm{~m}$ ) construit de torchis, les parois $E=0,10-0,18 \mathrm{~m}, \mathrm{H}=0,40$ m. Dans le foyer et sur la sole il $y$ avait des fragments céramiques de la dernière charge traitée. Le four est dérangé par une tombe d'époque médiévale. (Cronica sãpãturilor arheologice din Campania 1994, Cluj-Napoca, 1995, p. 66). Fig.8

Four no. 14: circulaire à pilier central (?) détruit pour la plupart. Le mur conservé $(E=0,37$ $\mathrm{m}$ ) construit de terre ménagée. La gueule, le pilier, la sole et le laboratoire sont détruits. (Cronica säpäturilor arheologice din campania 1994, Cluj-Napoca, 1995, p. 66).

Four no. 15: circulaire à pilier central (?) détruit presque entièrement. (Cronica sãpãturilor arheologice din campania 1997, Calarasi, 1998, p. 54).

Four no. 16: circulaire à pilier central désaffecté dès l'antiquité. $S=2,695 \mathrm{~m}^{2}$. Le foyer ( $\varnothing$ $=1,84 \mathrm{~m}$ ) construit de terre ménagée. La gueule orientée vers le $\mathrm{N}$ (canal commun avec C11). Pilier (fragmentaire, $\emptyset=0,16 \mathrm{~m}$ ) de terre ménagée. La sole (écroulée) construite de briques et de torchis comme liant. Le laboratoire n'est pas conservée. (Cronica sãpãturilor arheologice din campania 1996, Bucuresti, 1997, p. 43). 
Four no. 17: circulaire à pilier central. $S=1,767 \mathrm{~m}^{2}$. Le foyer (en bon état) fait en terre ménagée. La gueule n'a pas été saisie. Pilier en bon état, $H=0,45 \mathrm{~m}, \mathrm{E}=0,22 \mathrm{~m}$ ) La sole (fragmentaire) faite de briques à liant d'argile ( $\emptyset=1,10 \mathrm{~m}, E=0,12 \mathrm{~m}$ ). Le laboratoire (fragmentaire, $\emptyset=0,50 \mathrm{~m}$ ) en torchis, les parois $\mathrm{E}=0,10 \mathrm{~m}, \mathrm{H}$ conservée $=0,20 \mathrm{~m}$. (Cronica säpäturilor arheologice din campania 1997, Calarasi, 1998, p. 54)

Four no. 18: circulaire à pilier central, détruit dans la plus grande partie (information D. Elefterescu, inédit)

Four no. 19: rectangulaire à pilier central. Le foyer (fragmentaire) construit de terre ménagée $(\mathrm{L}=1,55 \mathrm{~m}, \mathrm{l}=1,45 \mathrm{~m}, \mathrm{H}=0,73 \mathrm{~m})$. La gueule orientée vers le $\mathrm{N}$. Les parois sont en terre ménagée. La sole (fragmentaire, $E=0,12 \mathrm{~m}, \mathrm{~L}=1,76 \mathrm{~m}, \mathrm{I}=1,48 \mathrm{~m}$ ) est faite de couches de torchis. Le laboratoire détruit pour la plupart, ses parois (conservées avec $\mathrm{H}=0,20-0,28 \mathrm{~m}$ ) sont en terre ménagée. Sur la sole on a trouvé des fragments céramique de la demière charge traitée.(information D. Elefterescu, inédit). Fig.10 

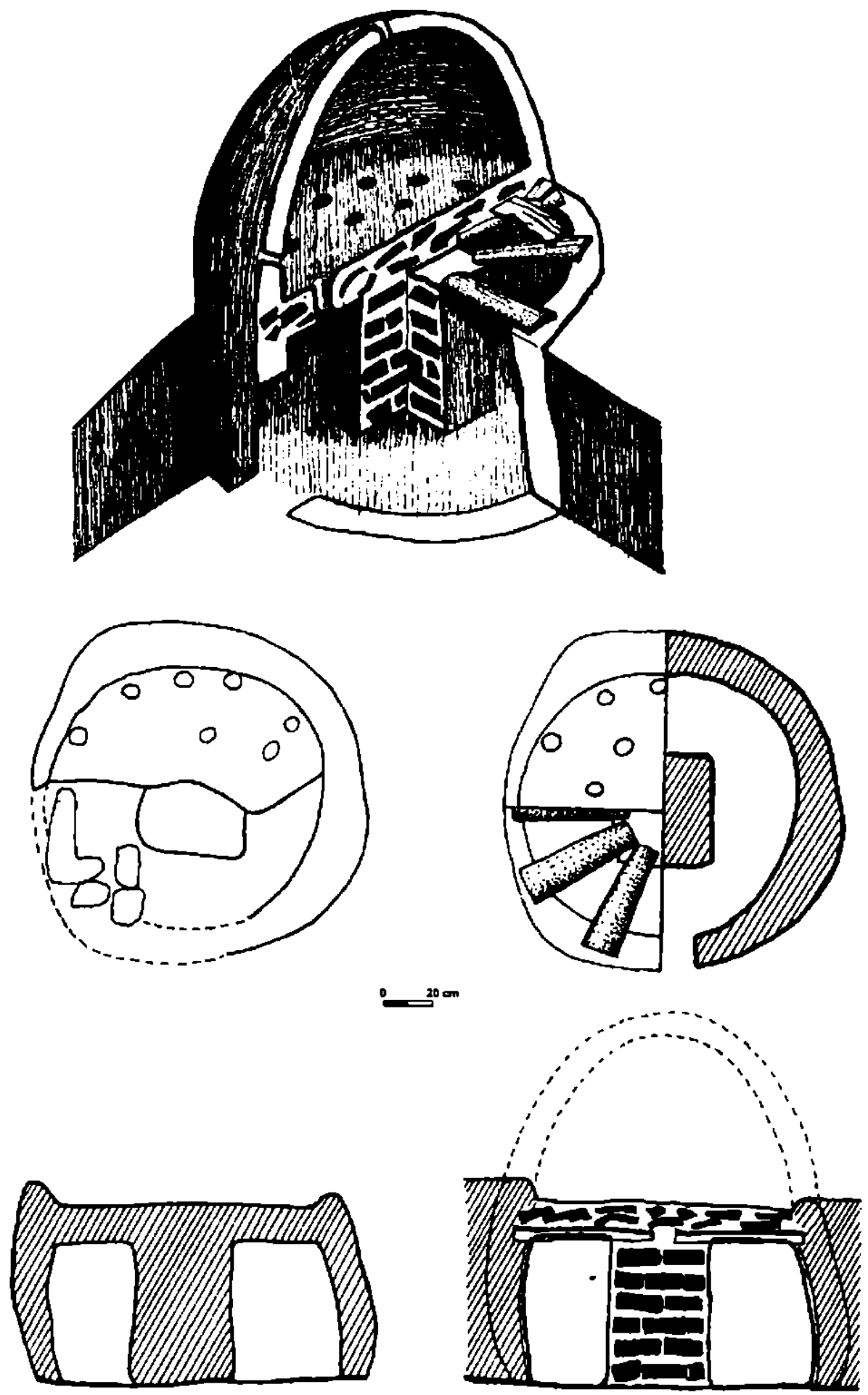

Fig. 9. Four 12 (reconstitution) 

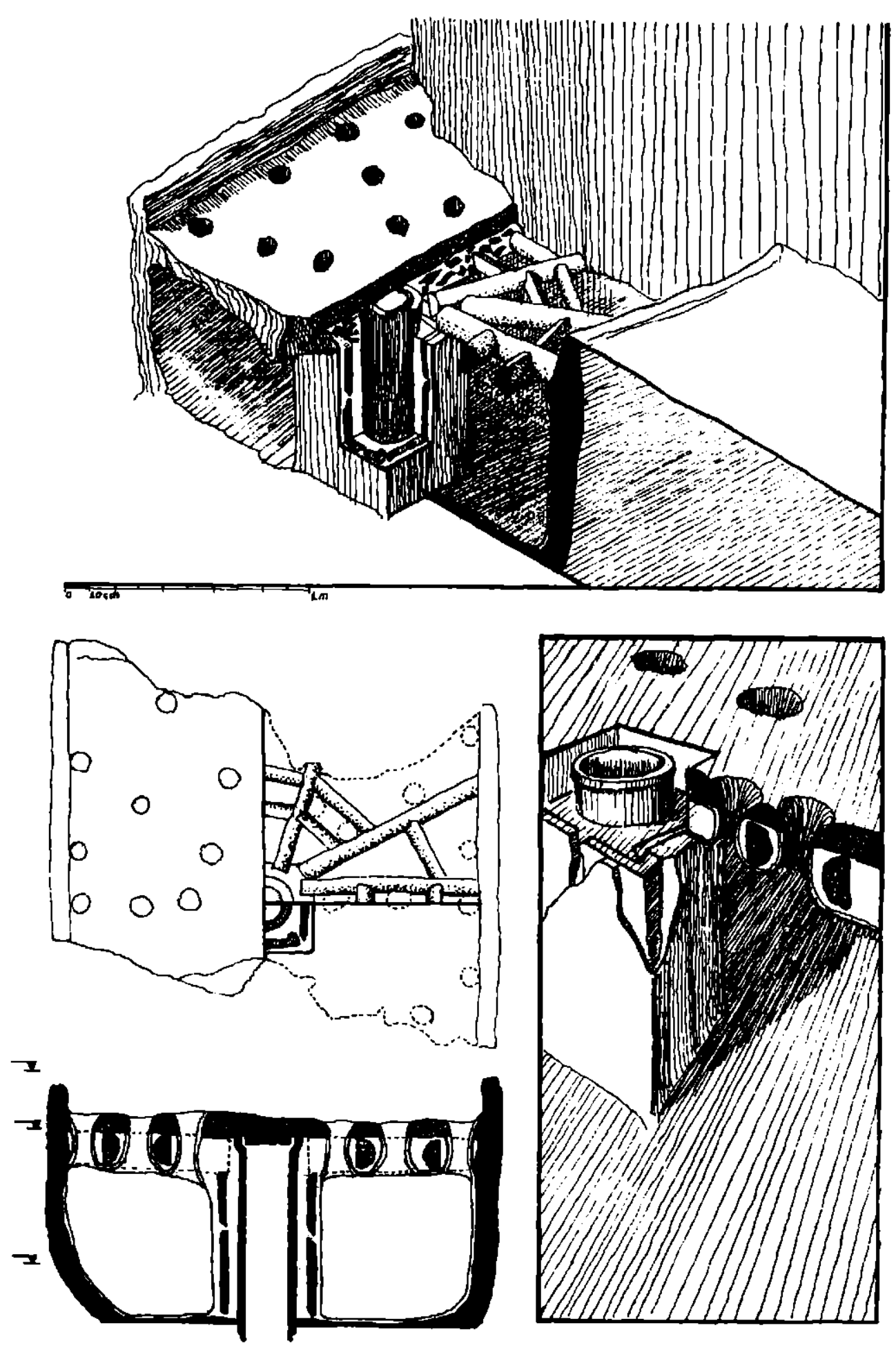

Fig. 10. Four 19 (reconstitution) 